\title{
Dissipative particle dynamics: A useful thermostat for equilibrium and nonequilibrium molecular dynamics simulations
}

\author{
Thomas Soddemann, ${ }^{*}$ Burkhard Dünweg, and Kurt Kremer \\ Max Planck Institute for Polymer Research, Ackermannweg 10, D-55128 Mainz, Germany
}

\begin{abstract}
We discuss dissipative particle dynamics as a thermostat to molecular dynamics, and highlight some of its virtues: (i) universal applicability irrespective of the interatomic potential; (ii) correct and unscreened reproduction of hydrodynamic correlations; (iii) stabilization of the numerical integration of the equations of motion; and (iv) the avoidance of a profile bias in boundary-driven nonequilibrium simulations of shear flow. Numerical results on a repulsive Lennard-Jones fluid illustrate our arguments.
\end{abstract}

\section{INTRODUCTION}

The natural thermodynamic ensemble of molecular dynamics (MD) simulations [1,2] is the microcanonical $N V E$ ensemble ( $N$ is the number of particles, $V$ is volume, and $E$ is energy). Nevertheless, in many cases one wants to modify the equations of motion such that the simulation runs in the canonical $N V T$ ensemble ( $T$ denoting the absolute temperature), i.e., to apply a so-called "thermostat" to the system. This may be desirable for various reasons listed below.

(i) In equilibrium situations, some thermodynamic relations (in particular fluctuation relations, e.g., for the specific heat) are often more straightforward to derive and to evaluate than for $N V E$.

(ii) The thermostat may tend to stabilize the simulation, such that a larger time step is permitted. This is true for Langevin-type stochastic thermostats (see below), and is a serious issue if a very long observation time is required. For example, when studying the dynamics of dense bead-spring polymer melts, one needs to observe the system over many millions of steps. Comparing the two simulations of (essentially) the same model, where one was run in the $N V E$ ensemble [3], using the Verlet algorithm [1,2], while the other [4] employed a stochastic dynamics (SD) Langevin thermostat [5], one sees that the $N V T$ ensemble permits a time step of $0.012 \tau$, while stability in the microcanonical ensemble requires a time step as small as $0.003 \tau$. Here $\tau$ denotes the natural time unit derived from the purely repulsive LennardJones potential to model the beads $[3,4]$.

(iii) In nonequilibrium molecular dynamics (NEMD) simulations [6] of steady states, the thermostat is of paramount importance. The system is driven by an external force, i.e., energy is pumped into the system and dissipated into heat. The thermostat is needed to remove this heat, just as in an experiment. It is, however, possible to combine the driving and thermostatting into one simple algorithm $[7,8]$, see below.

The present paper deals with one particular thermostat, the method of dissipative particle dynamics (DPD) [9-20]. It

\footnotetext{
*Present address: Rechenzentrum Garching, Boltzmannstraße 2, D-85748 Garching, Germany.
}

is a modification of the old SD thermostat, which keeps practically all of its virtues, while avoiding its most severe disadvantage - the lack of momentum conservation and concomitant incorrect reproduction of hydrodynamics, i.e., unphysical screening of hydrodynamic interactions [21]. Actually, DPD was originally developed in order to simulate fluids on a mesoscopic scale with correct hydrodynamic interactions. The idea was to use rather soft particles, which, vaguely spoken, should represent a cluster of atoms. This permits a large MD time step. Furthermore, a momentumconserving stochastic thermostat is added in order to model the internal degrees of freedom, which result in dissipation. While the original formulation [9] violated the fluctuationdissipation theorem, the more recent implementations based on the work by Español and Warren [11] satisfy it, and hence produce a well-defined $N V T$ ensemble. The thermostat thus allows even more increased time steps. However, it turned out rather soon that running these soft systems with very large time steps is less advantageous than originally expected: While the algorithm as such does remain stable, there are substantial discretization errors involved, such as that the measured temperature deviates significantly from the desired value. There have been many attempts to improve this situation by implementing more sophisticated integration schemes; this is currently a rather active field of research [15-20]. Interestingly enough, the time step issue is a very different one for hard potentials. In that case, the mere requirement of stability automatically enforces a rather small time step, under which condition the accuracy of the numerical solution is usually quite acceptable.

Unfortunately, many outlines of DPD discuss these two aspects (soft particles on one hand, thermostat on the other) as one unified method. However, as a matter of fact, they are completely independent, and thus it is perfectly legitimate to use the DPD thermostat also for simulations with "hard" particles. Such potentials are often desired in order to take molecular packing effects realistically into account, e.g., in the formation of mesophases of low molecular weight amphiphiles or in the study of entangled polymer systems. Though it was already stated in Ref. [13] that DPD can be viewed just as a thermostat to MD, the possibility to apply it to "hard" systems has not yet been widely exploited (exceptions are, e.g., Refs. [22,23]), and apparently its usefulness for such systems is not yet fully appreciated. The present 
paper is intended to fill this gap.

We run a standard MD system with an added DPD thermostat, thereby being able to afford a substantially larger time step compared to pure MD, and nevertheless reproducing hydrodynamic behavior correctly. This latter statement means, more precisely, that we correctly reproduce momentum propagation, which is often quite important in the dynamics of complex fluids. On the other hand, energy transport is not simulated faithfully, as the temperature is being kept constant on a local scale. Formally, this may be viewed as the limit of infinite thermal conductivity, which is not completely unrealistic, as for many systems the thermal conductivity is quite large. Furthermore, the "conventional" MD potentials force us to use a time step which is not too large (actually rather small in comparison with many DPD simulations of soft particles), and thus systematic discretization errors are of negligible importance for our simulations.

We have combined this approach with NEMD of shear flow, using a slight modification of the boundary-driven approach of Ref. [8]. We then arrive at an algorithm which is completely local. For parallelization, we use domain decomposition via a suitable adaptation of the method described in detail in Ref. [24].

The remainder of the paper is organized as follows. In Sec. II, we compare existing thermostats and NEMD schemes and state the arguments why we believe that our selected combination is useful. Section III discusses our simulational details, and presents a few test results. In particular, we study the shear viscosity of a simple liquid, comparing the SD to the DPD thermostat. Finally, we conclude in Sec. IV.

\section{THERMOSTATS AND NEMD ALGORITHMS}

\section{A. Thermostats}

There are several well-known MD thermostats which generate a well-defined $N V T$ ensemble. The Nosé-Hoover (NH) thermostat $[25,26]$ is a time-reversible deterministic scheme in which the system is coupled to one additional degree of freedom $\zeta$. In equilibrium, the equations of motion for an $N$-particle system in $d$-dimensional space are

$$
\begin{gathered}
\dot{\vec{r}}_{i}=\frac{\vec{p}_{i}}{m_{i}}, \\
\dot{\vec{p}}_{i}=\vec{F}_{i}-\zeta \vec{p}_{i}, \\
\dot{\zeta}=\frac{1}{M}\left[\sum_{i} \frac{\vec{p}_{i}^{2}}{m_{i}}-d N k_{B} T\right],
\end{gathered}
$$

where $\vec{r}_{i}$ are the particle coordinates, $\vec{p}_{i}$ the particle momenta, $m_{i}$ the masses, $\vec{F}_{i}$ the forces resulting from the interatomic potential, $k_{B}$ the Boltzmann constant, and $M$ a masslike parameter which sets the rate how quickly the system is thermostatted. In equilibrium, the variable $\zeta$ is Gaussian with zero mean and variance $\left\langle\zeta^{2}\right\rangle=k_{B} T / M$. Similarly, from the fluctuations of the kinetic energy one concludes that $\zeta$ has vanishing mean and variance $\left\langle\dot{\zeta}^{2}\right\rangle=2 d N\left(k_{B} T\right)^{2} / M^{2}$. This results in a typical time scale for the variation of $\zeta$ :

$$
\tau=\left(\frac{\left\langle\zeta^{2}\right\rangle}{\left\langle\dot{\zeta}^{2}\right\rangle}\right)^{1 / 2}=\left(\frac{M}{2 d N k_{B} T}\right)^{1 / 2}
$$

Efficient equilibration requires that this matches typical atomic time scales ("resonance"), i.e., $M \propto N$. In turn, this means that the typical $\zeta$ values scale like $N^{-1 / 2}$. In other words, the dynamics becomes more and more Newtonian when the system size is increased, and this means in turn that the method should reproduce hydrodynamics correctly if the system is chosen large enough. On the other hand, this also means that the $\mathrm{NH}$ thermostat does not stabilize the numerical integration of the equations of motion, because it is only based on a global feedback. Furthermore, the evaluation of the total kinetic energy involves global communication over all processors if the system is run on a parallel machine with domain decomposition. This is another disadvantage of the $\mathrm{NH}$ thermostat which should not be underestimated.

The SD thermostat [5] works quite differently. Here every particle is coupled to a viscous background and a stochastic heat bath, such that

$$
\begin{gathered}
\dot{\vec{r}}_{i}=\frac{\vec{p}_{i}}{m_{i}}, \\
\dot{\vec{p}}_{i}=\vec{F}_{i}-\zeta \frac{\vec{p}_{i}}{m_{i}}+\vec{f}_{i},
\end{gathered}
$$

where $\zeta$ is now a constant friction parameter, while the stochastic forces $\vec{f}_{i}$ have zero mean and satisfy the fluctuationdissipation theorem

$$
\left\langle f_{i}^{\alpha}(t) f_{j}^{\beta}\left(t^{\prime}\right)\right\rangle=2 \zeta k_{B} T \delta_{i j} \delta_{\alpha \beta} \delta\left(t-t^{\prime}\right),
$$

$\alpha$ and $\beta$ denoting Cartesian indices. The effect of this algorithm is to thermostat the system on a local scale. Particles which are too "cold" are given more energy by the noise term, while too "hot" particles are slowed down by the friction. Numerical instabilities, which usually arise from inaccurate calculation of a local collisionlike process, are thus effectively kept under control and cannot propagate. This is the reason why for this scheme a larger time step is possible than for pure MD. On the other hand, the algorithm violates Galilean invariance, as the damping biases the velocities towards the "laboratory" reference frame. This results in nonconservation of momentum (the center of mass of the overall system diffuses) and in effective damping of the hydrodynamic correlations on the length scale of a hydrodynamic screening length

$$
l=\left(\frac{\eta}{n \zeta}\right)^{1 / 2}
$$

where $\eta$ is the shear viscosity and $n$ the particle number density. This is seen quite straightforwardly by noticing that, in the hydrodynamic picture, the algorithm introduces a fric- 
tion force per unit volume $-\zeta n \vec{u}$, where $\vec{u}$ is the fluid streaming velocity. The random forces, on the other hand, are averaged to zero. Thus the term $\eta \Delta \vec{u}$ in the Stokes equation is replaced by $\eta \Delta \vec{u}-\zeta n \vec{u}$. Setting this to zero, and replacing $\Delta$ with $l^{-2}$, yields Eq. (5). For a more formal derivation, see Ref. [21]. For $\zeta \rightarrow 0$ the screening length diverges, as in this limit purely Newtonian dynamics is recovered.

The DPD algorithm is similar in spirit. There is also local friction and noise, such that the thermostatting and stabilizing features of SD are retained. As shown in Sec. III, we were able to run the DPD-thermostatted system with the same large time step as with SD. However, in contrast to SD, the friction does not dampen the "absolute" velocities of the particles, but rather the velocity differences of nearby particles. The method is thus sensitive to velocity gradients, as it should, in order to be consistent with hydrodynamics. Similarly, the stochastic forces act on pairs of nearby particles, such that Newton's third law is strictly fulfilled. The method thus satisfies the two basic requirements for reproducing hydrodynamics on large length and time scales: locality and momentum conservation. Indeed, it was shown formally that hydrodynamic behavior is recovered in that limit [12]. Care has to be taken to satisfy the fluctuationdissipation theorem to obtain a well-defined temperature. The original version of Hoogerbrugge and Koelman [9] did this incorrectly [it violated Eq. (12), see below]; the necessary modification was introduced by Español and Warren [11].

The DPD equations of motion are given by

$$
\begin{gathered}
\dot{\vec{r}}_{i}=\frac{\vec{p}_{i}}{m_{i}}, \\
\dot{\vec{p}}_{i}=\vec{F}_{i}+\vec{F}_{i}^{D}+\vec{F}_{i}^{R},
\end{gathered}
$$

where $\vec{F}_{i}^{D}$ denotes the additional damping (or dissipative) force on particle $i$ and $\vec{F}_{i}^{R}$ the corresponding random force. The latter are now based on particle pairs, i.e.,

$$
\begin{aligned}
& \vec{F}_{i}^{D}=\sum_{j(\neq i)} \vec{F}_{i j}^{D}, \\
& \vec{F}_{i}^{R}=\sum_{j(\neq i)} \vec{F}_{i j}^{R} .
\end{aligned}
$$

The dissipative force in the formulation of Español and Warren [11] is given by

$$
\vec{F}_{i j}^{D}=-\zeta w^{D}\left(r_{i j}\right)\left(\hat{r}_{i j} \cdot \vec{v}_{i j}\right) \hat{r}_{i j}
$$

and the random force by

$$
\vec{F}_{i j}^{R}=\sigma w^{R}\left(r_{i j}\right) \theta_{i j} \hat{r}_{i j} .
$$

Here, $\vec{v}_{i j}=\vec{v}_{i}-\vec{v}_{j}$ is the relative velocity between particles $i$ and $j$, while $\hat{r}_{i j}$ denotes the unit vector of the interatomic axis $\vec{r}_{i j}=\vec{r}_{i}-\vec{r}_{j} . \zeta$ is the friction constant and $\sigma$ the noise strength. $w^{D}$ and $w^{R}$ are $r$-dependent weight functions vanishing for $r \geqslant r_{c} . \theta_{i j}$ is a Gaussian white noise variable with $\theta_{i j}=\theta_{j i}$ and first and second moments

$$
\begin{gathered}
\left\langle\theta_{i j}(t)\right\rangle=0, \\
\left\langle\theta_{i j}(t) \theta_{k l}\left(t^{\prime}\right)\right\rangle=\left(\delta_{i k} \delta_{j l}+\delta_{i l} \delta_{j k}\right) \delta\left(t-t^{\prime}\right) .
\end{gathered}
$$

$\vec{F}^{D}$ and $\vec{F}^{R}$ act along the interatomic axis and thus conserve the momentum. There is an independent random function for each pair of particles. In order to satisfy the fluctuationdissipation theorem, the relations

$$
\sigma^{2}=2 k_{B} T \zeta
$$

and

$$
\left[w^{R}(r)\right]^{2}=w^{D}(r)
$$

must hold [11]. The usual choice is

$$
w^{D}(r)=\left[w^{R}(r)\right]^{2}=\left\{\begin{array}{l}
\left(1-r / r_{c}\right)^{2}, \quad r<r_{c} \\
0, \quad r \geqslant r_{c} .
\end{array}\right.
$$

Another possible choice, which might be computationally more efficient, would be

$$
w^{D}(r)=w^{R}(r)= \begin{cases}1, & r<r_{c} \\ 0, & r \geqslant r_{c}\end{cases}
$$

The scheme is thus seen to combine the positive aspects of the two previous thermostats: Strict Galilean invariance and correct hydrodynamics, as $\mathrm{NH}$ for large systems, and numerical stabilization, as SD. We hence believe it to be the ideal thermostat whenever one studies problems where hydrodynamics (momentum transport, but not heat flow) is (or is suspected to be) important.

Yet another approach for thermostatting has been suggested by Andersen [27]. Instead of solving a Langevin equation, the procedure periodically picks some particle at random and assigns a new random velocity from a Maxwell distribution to it. This procedure generates a canonical distribution, and, like SD, it does not conserve the momentum. Unlike SD, however, it does not "smear out" the thermostatting homogeneously (with respect to both space and time), but rather generates kicks which are localized and rather strong (the trajectory is not continuous in phase space). This is a property which we view as somewhat disadvantageous compared to SD (and this is why we do not use it); nevertheless, in many cases the method has been applied very successfully. The Andersen method is also computationally slightly more efficient than SD, since only now and then a single particle is involved.

In the same way as DPD can be viewed as the momentum-conserving version of $\mathrm{SD}$, one can also devise a momentum-conserving version of the Andersen thermostat, which works along similar lines as DPD (again, relative velocities are thermostatted). This idea has been put forward by 
Lowe [28]. Concerning the comparison with DPD, the same comments can be made as for the comparison of SD vs Andersen.

\section{B. NEMD algorithms for shear}

A simple way to introduce a shear rate

$$
\dot{\gamma}=\frac{\partial u_{x}}{\partial y}
$$

with $u_{y}=u_{z}=0$ for simple Couette flow is by modifying the periodic boundary conditions (Lees-Edwards boundary conditions [29]). A particle that leaves the box in $y$ direction at the "top" and reenters at the "bottom" is displaced appropriately both in position and velocity space. Furthermore, a thermostat must be added in order to remove the viscous heat; this shall be discussed below. It should be noted that this scheme explicitly breaks the translational invariance in $y$ direction: The positions where "something happens" to the particles are well-defined layers in space. Hence, the method can be viewed as a boundary-driven method. Another popular approach is to use the so-called Sllod equations of motion [6] (so named because of its close relationship to the Dolls tensor algorithm). This is a homogeneous (or "synthetic") method, where the effect of the imposed shear is rather smeared out homogeneously over the $y$ axis, and a linear shear profile is enforced (i.e., translational invariance is reestablished).

Boundary-driven and homogeneous methods have both advantages and disadvantages. The advantage of a homogeneous method is that there are no corrections to the bulk behavior by boundary layers, such that rather small systems can be studied. Homogeneous methods are therefore very well suited for the efficient calculation of linear transport coefficients (although some care must be taken to verify that the simulation is indeed in the linear regime). The disadvantage is that the linear profile is enforced such that the applicability is restricted to cases where the profile indeed is linear. This, however, is not always the case. Many complex fluids exhibit the phenomenon of "shear banding," where the translational symmetry in $y$ direction is spontaneously broken as a result of a hydrodynamic instability. A homogeneous algorithm suppresses the occurrence of such instabilities, and can therefore produce incorrect physics. Conversely, a boundary-driven method allows the system to choose its own profile (if the thermostat does so, too), and is hence able to study such phenomena. It must, however, be noted that boundary-driven approaches tend to require larger systems. Nevertheless, as the emphasis of present day simulations is more on nonlinear phenomena, we think that boundarydriven methods are preferable, and therefore we will not discuss homogeneous methods any further.

Let us now discuss the thermostat. Sticking to the "boundary-driven" philosophy, it is obviously the "cleanest" way to restrict the thermostatting to boundary layers, too, while the interior of the sample is run with pure $N V E$ dynamics. This implies cooling of the layers, and some heat flows from the center (where the viscous heat is produced) to the layers. A particularly simple and ingenious way to restrict driving and thermostatting to boundary layers in one common algorithm has recently been put forward by MüllerPlathe (for heat transport in Ref. [7], for shear simulations in Ref. [8]). One regularly selects pairs of particles with the property that they reside in opposite layers, and that their velocity differs particularly strongly from the desired velocity of the layer. Then the velocities are just exchanged. In case of a multicomponent fluid, one has to take care that the pairs are selected in a way that the masses are identical. This obviously conserves momentum and energy, and hence produces a stable steady state, such that no additional thermostatting is necessary. Surely enough, the cooling at the boundaries is indeed observed [8]. The easiest way to understand this is to note that viscous heating is nothing but entropy production and that the algorithm actually removes entropy at the layers by artificially putting in information (in essence, the method is just a Maxwell demon). The viscosity then results directly from the ratio between transported momentum (or applied force) and resulting shear gradient.

A slight technical difficulty arises with this algorithm when trying to apply it to small systems which are only weakly sheared. This is particularly true when one attempts to control the shear rate from the outset by a feedback procedure which enforces velocity exchanges from the criterion of the momentary shear being too small or too large. The simplistic procedure to select within a layer just the particle whose velocity differs most strongly from the desired layer velocity may lead to large overshoots of the layer velocity after the exchange, since even a single-particle exchange already may give a momentum transfer which is significantly too large. This requires to either correct this in the subsequent step, which results in undesirable oscillations, or to carefully select the pair for exchange to reach the desired result. In the latter case, a rather cumbersome search procedure is necessary.

On the other hand, if the system is thermostatted in the bulk (in order to stabilize the integration of the equations of motion and to enforce a homogeneous temperature profile), it is not necessary to drive the system via a Maxwell demon. This can rather be done by simply applying a uniform force on all the layer particles, which is adjusted every single time step to keep the shear rate strictly constant. It is this latter procedure which we have implemented in our tests, which we restricted to a rather small system of only 4096 particles. Here we used simple periodic boundary conditions in all three directions for a box of size $L_{x} \times L_{y} \times L_{z}$. Two thin layers perpendicular to the $y$ axis, with distance $L_{y} / 2$, were chosen for driving in the $+x$ and $-x$ direction, respectively. This setup effectively generates two Couette cells with opposite shear gradients. Compared to Lees-Edwards boundary conditions, this procedure has the advantage that the driving occurs only in velocity space, such that it is quite readily implemented as a modification of a parallelized equilibrium simulation. In our case, we used a straightforward adaptation of the program described in Ref. [24].

An important issue of thermostatting the system in the bulk is that one has to make sure that a so-called profileunbiased thermostat (PUT) [6] is applied. For NH, one de- 
fines the so-called "peculiar velocities" $\vec{c}_{i}[6]$ as the difference between the actual velocities and the expected linear profile. These are used to define the kinetic temperature in the equation of motion for $\zeta$. However, this scheme is a typical example of a profile-biased thermostat (PBT) [6]. While the procedure is perfectly legitimate in the linearresponse regime (here the profile is linear anyways), unphysical results must be expected (and have been observed, see Ref. [6]) in the nonlinear regime: The PBT prefers the linear profile and thus tends to suppress the occurrence of hydrodynamic instabilities. The way out is to use a PUT which does not single out a prescribed profile but rather allows the system choose its own. To do this within the framework of NH is possible, but implies a rather awkward selfconsistent procedure [6].

For SD, a possible choice is to only thermostat the velocities in $y$ and $z$ direction (recall $u_{y}=u_{z}=0$ for Couette flow). This should not pose any problem as long as one studies simple shear in the linear regime. However, in the nonlinear regime such a procedure is somewhat dangerous as it presupposes a certain symmetry of the steady state, which may be broken (and usually the kind of symmetry breaking is not known in advance). For simple shear banding, where only the translational invariance in $y$ direction is broken, the procedure is probably acceptable. The most naive approach, i.e., to just apply the SD thermostat in all three directions, will fail even in the linear regime and produce an incorrect apparent viscosity; this is outlined in the Appendix. The same is true if one thinks of thermostatting the peculiar velocities; in this case the "friction" term $-\zeta \vec{c}_{i}$ would punish any velocity which is not in accord with the prescribed velocity profile, and actually drive the system in the bulk, which is clearly not desired.

Conversely, the DPD thermostat, which is based upon relative velocities, does not presuppose any sort of symmetry, and is profile unbiased by construction.

Taking all these considerations together, we thus arrive at what we believe to be a very suitable algorithm to study nonlinear effects in shear flow: Use a boundary-driven method combined with the DPD thermostat. This results in a simple and easy-to-implement simulation with a straightforward PUT, which keeps the temperature profile constant, and, as an additional bonus, stabilizes the integration of the equations of motion. If there is suspicion that heat flow might be important, one can instead avoid thermostatting altogether and drive the system by a Maxwell demon.

In order to demonstrate that it is really important to allow the system to choose the profile of its own liking, we show in Fig. 1 the configuration of a system of amphiphilic molecules, simulated by the model outlined in Ref. [30], using essentially the algorithm described above [31]. The system exhibits very strong shear banding: While the ordered regions move essentially as "blocks," the shear is concentrated in the narrow strips where it is disordered. More details of this simulation will be published elsewhere.

\section{SIMULATIONS}

The simulations were carried out with a system consisting of 4096 Lennard-Jones (LJ) particles at a density of $\rho$

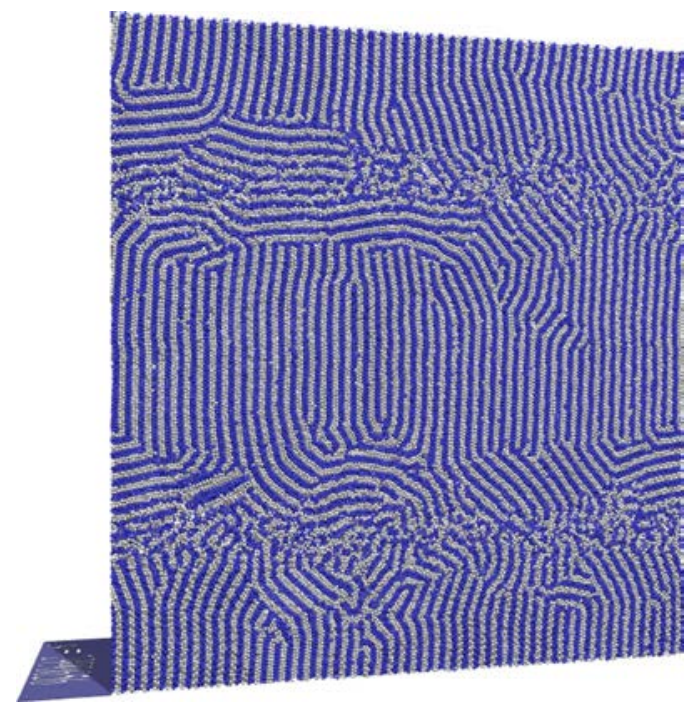

FIG. 1. Shear-banded state of a large system of amphiphilic molecules modeled as $995328 A-B$ dimers with attractive $A-A$ and $B-B$ interactions. The particle color is used to distinguish between $A$ and $B$. Direction of view is the $x$ direction, i.e., the direction of the shear velocity. Direction up-down is the $y$ direction, i.e., the direction of the shear gradient. The system organizes in lamellas whose normal is oriented in $z$ direction. The driving occurs at narrow layers at (i) the top/bottom and (ii) the center of the box. Near these layers, the velocity gradient is essentially zero, and the molecules move as a homogeneous "block." The shear gradient is concentrated in small regions located in the middle between the driving layers; in these regions the system is disordered.

$=0.85$ (in standard reduced units where the particle mass as well as the LJ parameters $\epsilon$ and $\sigma$ are set to unity) in a cubic box with periodic boundary conditions. The LJ potential was cut off at a separation $r_{c}=2^{1 / 6}$ and shifted, so that only its repulsive part is left. The thermostatting temperature was set to $k_{B} T=1$. For the thermostats (both SD and DPD) uniform random numbers were used, since it has been shown that they are just as good as Gaussian ones for Langevin simulations [32]. For the weight functions of the DPD thermostat, we used the standard choice [Eq. (13)] and $r_{c}=2^{1 / 6}$ for the cutoff. We integrated the equations of motions with the velocity Verlet algorithm [1,2], using a time step $\Delta t=0.01$ both for SD and DPD. As already mentioned, this is large compared to strict Newtonian MD, while small compared to DPD simulations with ultrasoft particles and ultralarge time steps. Therefore, our simulation was not hampered by the typical large discretization errors of large time-step DPD simulations $[15-20]$. To test this, we measured the temperature in equilibrium and found it to converge from a high value of $k_{B} T=10$ to the simulation temperature $k_{B} T=1$ within 400 time steps for DPD in comparison to somewhat less than 600 for SD, at a damping constant of $\zeta=1$. The usual choice for SD lies in the range $0.5 \leqslant \zeta \leqslant 1.5$; this ensures that the friction from the algorithm is still rather small compared to the intrinsic friction from the surrounding particles for these dense systems.

We did not carefully analyze the equilibrium properties of the fluid, since they are essentially known from previous 


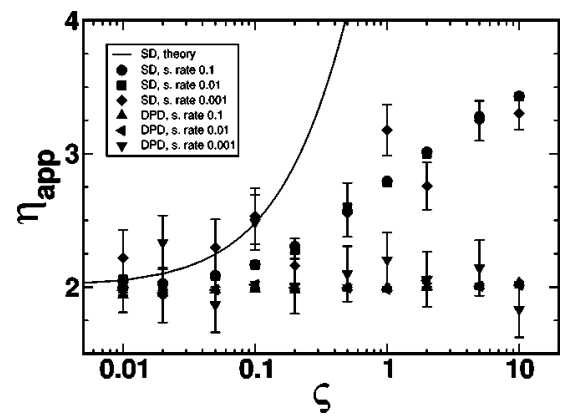

FIG. 2. Apparent shear viscosity $\eta_{a p p}$ as a function of the friction constant $\zeta$ for different shear rates and thermostats, as indicated in the plot. The line indicates the theoretical prediction [Eq. (16)].

simulations: In Ref. [33] the same model was studied in detail, however at a slightly different state point $(\rho=0.864$, $\left.k_{B} T=1.2\right)$. Taking the results from that simulation, we know that our fluid is characterized by (i) a highly structured pair correlation function $g(r)$, (ii) a large pressure $P \approx 10$, (iii) a viscosity $\eta \approx 2$, and similarly (iv) a kinematic viscosity $\nu$ $\approx 2$, while $(\mathrm{v})$ the particle diffusion constant $D$ is roughly $D \approx 0.07$. From this, one sees that the Schmidt number $\mathrm{Sc}$ $=\nu / D$ is roughly $\mathrm{Sc} \approx 30$, which is a reasonable value for real fluids (large Sc means that diffusive momentum transport is substantially faster than mass transport). For more details, see Ref. [33].

Concerning computational efficiency, it is obvious that DPD is somewhat more expensive than SD, since it involves the calculation of velocity differences and of unit vectors, plus the generation of substantially more random numbers. In our simulations, we found an average slowdown of $35 \%$. Note that one could optimize the DPD procedure further by (i) introducing the simpler weight function [Eq. (14)] and (ii) applying friction and noise not every single time step, but, say, every second or third step (pushing this idea to its limits, one would arrive at the Lowe-Andersen [28] thermostat).

In Fig. 2, we compare the apparent viscosity $\eta_{a p p}$, as obtained from naive SD and from DPD runs, varying the friction coefficient $\zeta$ over a substantial range. The data show, on one hand, that for shear rates $\dot{\gamma}=0.001,0.01,0.1$ there is not yet a measurable dependence on $\dot{\gamma}$ and, on the other hand, that $\eta_{a p p}$ is independent of the friction coefficient only for DPD but not for SD. In the latter case, there is a sizable increase with $\zeta$, and the physical value $\eta_{0}$ is only recovered in the limit $\zeta \rightarrow 0$. The theoretical considerations of the Appendix explain this increase as a result of hydrodynamic screening, which gives rise to an inhomogeneous shear profile which is concentrated around the driving layers, with thickness of the order of the hydrodynamic screening length $\kappa^{-1}=l$ [see Eq. (5)]. The resulting prediction

$$
\frac{\eta_{\text {app }}}{\eta_{0}}=\frac{\kappa L_{y}}{4} \operatorname{coth}\left(\frac{\kappa L_{y}}{4}\right)=1+\frac{1}{3}\left(\frac{\kappa L_{y}}{4}\right)^{2}+O\left(\kappa^{4}\right)
$$

is also shown in Fig. 2, using the value $\eta_{0}=2$. Obviously, the increase in $\eta_{a p p}$ is much weaker than expected. We do not fully understand this deviation but believe that it is some sort of finite size effect. The system has a thickness of the order of 16 atomic layers, and this is probably not enough to faithfully represent the strongly modified profile. Indeed, it is reasonable to assume that the atomic structure of the fluid prohibits the decrease of the screening length below a value of the order of a particle diameter. If we thus assume $l_{\min }$ $=2$ or $\kappa_{\max }=0.5$, we find that $\eta_{a p p} / \eta_{0}$ cannot exceed the value $\kappa_{\max } L_{y} / 4 \approx 2$, which is roughly what we observe.

Strictly speaking, for the DPD case a constant $\eta_{a p p}$ is not expected either. Rather the theoretical prediction is [12]

$$
\frac{\eta_{a p p}}{\eta_{0}}=1+O\left(\zeta^{2}\right)
$$

The prefactor of the correction term is nonuniversal and can be written as a Green-Kubo integral over the autocorrelation function of the dissipative stresses. The important point, however, is that (in contrast to SD) it is an intensive quantity, i.e., does not depend on the system size $L_{y}$. Indeed the data of Fig. 2 show no systematic increase of $\eta_{a p p}$ within our range of $\zeta$, and within our error bars. For our system, whose behavior is dominated by the hard interatomic interactions, the correction due to the friction is below resolution within the studied range of $\zeta$ values.

\section{CONCLUSIONS}

Our considerations and test results show that DPD is a very useful thermostat for MD, which should be used whenever hydrodynamics (momentum transport) is important. Unlike SD, it does not screen the hydrodynamic correlations and, unlike NH, it is completely local. To best of our knowledge, it is the first thermostat which avoids profile biasing of NEMD simulations in a very natural and simple way, as it introduces neither an absolute reference frame (as SD) nor the concept of "peculiar velocities" (as NH). We think that it is therefore the ideal thermostat for NEMD simulationswith the caveat that its applicability is of course restricted to phenomena where energy transport plays no role (for example, it would not be applicable for studying, say, Rayleigh-Benard convection). In such cases, strictly Newtonian MD, combined with a Maxwell demon along the lines of Refs. [7,8], is most probably the method of choice. These considerations are all in accord with our general belief that nonlinear phenomena in nonequilibrium systems should be studied by methods which do not interfere with the system in the bulk. From this perspective, the main advantage of DPD compared to just Newtonian MD is the stabilization of the numerical integration scheme, which is also very important, in particular for simulations with long observation times.

\section{ACKNOWLEDGMENTS}

We thank F. Müller-Plathe, J. Vollmer, H. Pleiner, H. Brand, and G. Auernhammer for fruitful discussions.

\section{APPENDIX: PROFILE BIASING AND APPARENT VISCOSITY FOR THE LANGEVIN THERMOSTAT}

In the boundary-driven method, the apparent viscosity is obtained by measuring the average force $F$ (momentum 
transfer per unit time) exerted onto the boundary layers and normalizing it by their area $L_{x} L_{z}$ and the shear gradient $\dot{\gamma}$ $=\partial u_{x} / \partial y$ :

$$
\eta=F /\left(\dot{\gamma} L_{x} L_{z}\right) .
$$

Here we have assumed an $L_{x} \times L_{y} \times L_{z}$ simulation cell and a shear gradient in $y$ direction, while the velocity flow field is in $x$ direction. This procedure is, by definition, correct if the underlying dynamics in the bulk is Newtonian (i.e., thermostatting occurs only at the boundaries, too) and $\dot{\gamma}$ is small enough to exclude nonlinear effects. An equivalent procedure is obtained by realizing that the average dissipated energy per unit time and unit volume is given by $\eta \dot{\gamma}^{2}$, resulting in a total dissipated power of $\eta L_{x} L_{y} L_{z} \dot{\gamma}^{2}$. On the other hand, the power put into the system by external driving is $F u$ $=F \dot{\gamma} L_{y}$. By equating these expressions, one again obtains Eq. (A1).

For a system which is subject to a bulk Langevin thermostat, the apparent viscosity $\eta_{a p p}$, as measured by this procedure, will in general differ from the true viscosity $\eta_{0}$. Since the produced velocity profile $u(y)$ will in general not be linear, we generalize the above consideration to yield

$$
\frac{\eta_{a p p}}{\eta_{0}}=\frac{1}{\eta_{0} L_{y} \dot{\gamma}^{2}} \int_{-L_{y} / 2}^{+L_{y} / 2} d y\left\{\eta_{0}\left(\frac{d u}{d y}\right)^{2}+P(u(y))\right\} .
$$

Here we assume that the simulation cell extends from $-L_{y} / 2$ to $+L_{y} / 2$, while $P$ denotes the average energy per unit time and unit volume which is dissipated by the thermostat. Following a general principle of linear nonequilibrium thermodynamics, we now assume that $u$ will adjust in such a way that the above energy dissipation rate will be minimum.

Further progress requires calculation of $P$. The Langevin equation for a single particle with mass $m$ (all particles are assumed to have identical mass) is written as

$$
m \frac{d}{d t} \vec{v}_{i}=\vec{F}_{i}-\zeta \vec{v}_{i}+\vec{f}_{i}
$$

where the stochastic forces have zero mean and satisfy the fluctuation-dissipation theorem

$$
\left\langle f_{i}^{\alpha}(t) f_{j}^{\beta}\left(t^{\prime}\right)\right\rangle=2 \zeta k_{B} T \delta_{i j} \delta_{\alpha \beta} \delta\left(t-t^{\prime}\right) .
$$

Therefore the average dissipation power by the friction term is given by

$$
P_{f r}=n \zeta\left\langle\vec{v}_{i}^{2}\right\rangle,
$$

where $n$ denotes the particle density needed to transform from dissipation per particle to dissipation per unit volume. We now decompose the velocity into the flow velocity and the peculiar velocity, writing $\vec{v}_{i}=\vec{u}+\vec{c}_{i}$, and note that for weak driving the variance in terms of peculiar velocities is still given by $\left\langle\vec{c}_{i}^{2}\right\rangle=3 k_{B} T / m$, as in the equilibrium case. We hence find

$$
P_{f r}=n \zeta\left[\vec{u}^{2}+3 k_{B} T / m\right] .
$$

Similarly, it is straightforward to show that the random displacements in velocity space result in an average increase of the kinetic energy, resulting in a term $P_{s t}=-3 n \zeta k_{B} T / m$. Therefore, the total dissipative power per unit volume is

$$
P=n \zeta u^{2},
$$

where we have assumed that $\vec{u}$ points in $x$ direction. In the equilibrium case $u=0, P$ vanishes, as it should be. Equation (A2) thus becomes

$$
\frac{\eta_{a p p}}{\eta_{0}}=\frac{1}{\eta_{0} L_{y} \dot{\gamma}^{2}} \int_{-L_{y} / 2}^{+L_{y} / 2} d y\left\{\eta_{0}\left(\frac{d u}{d y}\right)^{2}+n \zeta u^{2}\right\} .
$$

We now introduce reduced variables $\lambda$ and $\phi$ by writing $y$ $=L_{y} \lambda /(2 \pi)$ and $u=\dot{\gamma} L_{y} \phi / 4$, and the screening parameter $\kappa^{2}=n \zeta / \eta_{0}\left(\kappa^{-1}\right.$ is just the hydrodynamic screening length of the algorithm [21]). This transforms Eq. (A8) to

$$
\frac{\eta_{a p p}}{\eta_{0}}=\int_{-\pi}^{+\pi} d \lambda\left\{\frac{\pi}{8}\left(\frac{d \phi}{d \lambda}\right)^{2}+\frac{1}{2 \pi}\left(\frac{\kappa L_{y}}{4}\right)^{2} \phi^{2}\right\} .
$$

We now turn to the minimization of this expression, taking into account the way in which the simulation is run. First, we have periodic boundary conditions in all three spatial directions, which allows us to write the profile in terms of a Fourier expansion as

$$
\phi(\lambda)=b_{0}+\sum_{n=1}^{\infty}\left\{a_{n} \sin (\lambda n)+b_{n} \cos (\lambda n)\right\} .
$$

Inserting this expression into Eq. (A9), one finds after some straightforward algebra

$$
\frac{\eta_{a p p}}{\eta_{0}}=\left(\frac{\kappa L_{y}}{4}\right)^{2} b_{0}^{2}+\frac{1}{2} \sum_{n=1}^{\infty} C_{n}\left(a_{n}^{2}+b_{n}^{2}\right)
$$

with

$$
C_{n}=\frac{1}{4} \pi^{2} n^{2}+\left(\frac{\kappa L_{y}}{4}\right)^{2}
$$

Second, the shear is imposed in the layers $y= \pm L_{y} / 4$, such that $u\left(L_{y} / 4\right)-u\left(-L_{y} / 4\right)=\dot{\gamma} L_{y} / 2$ or

$$
\sum_{p=0}^{\infty} a_{2 p+1}(-1)^{p}=1 .
$$

Minimizing the dissipation rate with the constraint (A13), one finds that all coefficients except $a_{1}, a_{3}, \ldots$ vanish. The nonvanishing coefficients are given by

$$
a_{2 p+1}=\frac{2(-1)^{p}}{C_{2 p+1}} \frac{\kappa L_{y}}{4} \operatorname{coth}\left(\frac{\kappa L_{y}}{4}\right) ;
$$

here we have made use of the relation [34] 


$$
\sum_{p=0}^{\infty} \frac{1}{C_{2 p+1}}=\frac{1}{2} \frac{4}{\kappa L_{y}} \tanh \left(\frac{\kappa L_{y}}{4}\right) .
$$

Inserting this solution into Eq. (A11), and using Eq. (A15) again, one finds

$$
\frac{\eta_{a p p}}{\eta_{0}}=\frac{\kappa L_{y}}{4} \operatorname{coth}\left(\frac{\kappa L_{y}}{4}\right)=1+\frac{1}{3}\left(\frac{\kappa L_{y}}{4}\right)^{2}+O\left(\kappa^{4}\right) .
$$

The profile can also be obtained as a closed expression by noting that the Euler-Lagrange equation corresponding to functional (A9) is given by the modified Stokes equation

$$
\frac{\pi}{4} \frac{d^{2} \phi}{d \lambda^{2}}=\frac{1}{\pi}\left(\frac{\kappa L_{y}}{4}\right)^{2} \phi
$$

with solution (between $\lambda=-\pi / 2$ and $+\pi / 2$ )

$$
\phi(\lambda)=\frac{\sinh \left(\frac{\kappa L_{y}}{2 \pi} \lambda\right)}{\sinh \left(\frac{\kappa L_{y}}{4}\right)}
$$

[note that $\phi$ must be odd and $\phi(\pi / 2)=1$ ]. This implies cusps at $\lambda= \pm \pi / 2$. It is straightforward to check that the Fourier coefficients of this profile are indeed given by Eq. (A14). One also sees that in the Newtonian limit $\kappa=0$, a sawtooth profile is recovered. It should be noted that quite analogous considerations have already been put forward in Ref. [35].

The important point about this reasoning is that the modifications become arbitrarily large when the system size $L_{y}$ increases. Indeed, in the limit $L_{y} \rightarrow \infty$, we just have

$$
\frac{\eta_{a p p}}{\eta_{0}}=\frac{\kappa L_{y}}{4} .
$$

Furthermore, one sees from Eq. (A18) that the shear is concentrated in a small layer, whose size is given by the hydrodynamic screening length $\kappa^{-1}$. In other words, the hydrodynamic screening prevents the driving at the boundaries from having any effect beyond that layer. The data analysis therefore underestimates the shear gradient by a factor of order $\kappa^{-1} / L_{y}$, i.e., overestimates the viscosity by a factor of order $\kappa L_{y}$. This is the physical interpretation of Eq. (A19).
[1] M.P. Allen and D.J. Tildesley, Computer Simulation of Liquids (Clarendon, Oxford, 1987).

[2] D.C. Rapaport, The Art of Molecular Dynamics Simulation (Cambridge University Press, New York, 1995).

[3] A. Kopf, B. Dünweg, and W. Paul, J. Chem. Phys. 107, 6945 (1997).

[4] M. Pütz, K. Kremer, and G.S. Grest, Europhys. Lett. 49, 735 (2000).

[5] T. Schneider and E. Stoll, Phys. Rev. B 17, 1302 (1978).

[6] D. Evans and G. Morriss, Statistical Mechanics of Nonequilibrium Liquids (Academic Press, London, 1990).

[7] F. Müller-Plathe, J. Chem. Phys. 106, 6082 (1997).

[8] F. Müller-Plathe, Phys. Rev. E 59, 4894 (1999).

[9] P.J. Hoogerbrugge and J.M.V.A. Koelman, Europhys. Lett. 19, 155 (1992).

[10] J.M.V.A. Koelman and P.J. Hoogerbrugge, Europhys. Lett. 21, 369 (1993).

[11] P. Español and P. Warren, Europhys. Lett. 30, 191 (1995).

[12] P. Español, Phys. Rev. E 52, 1734 (1995).

[13] R. Groot and P. Warren, J. Chem. Phys. 107, 4423 (1997).

[14] P. Español, Phys. Rev. E 57, 2930 (1998).

[15] I. Pagonabarraga, M.J.H. Hagen, and D. Frenkel, Europhys. Lett. 42, 377 (1998).

[16] J.B. Gibson, K. Chen, and S. Chynoweth, Int. J. Mod. Phys. C 10, 241 (1999).

[17] G. Besold, I. Vattulainen, M. Karttunen, and J.M. Polson,
Phys. Rev. E 62, R7611 (2000).

[18] I. Vattulainen, M. Karttunen, G. Besold, and J.M. Polson, J. Chem. Phys. 116, 3967 (2002).

[19] P. Nikunen, M. Karttunen, and I. Vattulainen, Comput. Phys. Commun. 153, 407 (2003).

[20] T. Shardlow, SIAM J. Sci. Comput. (USA) 24, 1267 (2003).

[21] B. Dünweg, J. Chem. Phys. 99, 6977 (1993).

[22] W. Dzwinel and D.A. Yuen, J. Colloid Interface Sci. 225, 179 (2000).

[23] W. Dzwinel and D.A. Yuen, Int. J. Mod. Phys. C 11, 1 (2000).

[24] M. Pütz and A. Kolb, Comput. Phys. Commun. 113, 145 (1998).

[25] S. Nosé, J. Chem. Phys. 81, 511 (1984).

[26] W.G. Hoover, Phys. Rev. A 31, 1695 (1985).

[27] H. Andersen, J. Chem. Phys. 72, 2384 (1980).

[28] C.P. Lowe, Europhys. Lett. 47, 145 (1999).

[29] A.W. Lees and S.F. Edwards, J. Phys. C 15, 1921 (1972).

[30] T. Soddemann, B. Dünweg, and K. Kremer, Eur. Phys. J. E 6, 409 (2001).

[31] T. Soddemann, Ph.D. thesis, University of Mainz, 2001.

[32] B. Dünweg and W. Paul, Int. J. Mod. Phys. C 2, 817 (1991).

[33] B. Dünweg and K. Kremer, J. Chem. Phys. 99, 6983 (1993).

[34] I.S. Gradshteyn and I.M. Ryzhik, Table of Integrals, Series, and Products (Academic Press, San Diego, 1980).

[35] T. Kreer, M.H. Müser, K. Binder, and J. Klein, Langmuir 17, 7804 (2001). 